\title{
Solution of Five-dimensional Schrodinger equation for Kratzer's potential and trigonometric tangent squared potential with asymptotic iteration method (AIM)
}

\author{
Agung Budi Prakoso ${ }^{1}$, A Suparmi ${ }^{2}, \mathrm{C} \mathrm{Cari}^{2}$ \\ ${ }^{1,2}$ Physics Department, Faculty of Science and Mathematics, Universitas Sebelas Maret \\ Jl. Ir. Sutami 36A Kentingan Jebres Surakarta 57126 \\ E-mail: agungbudi12101993@student.uns.ac.id
}

Received 28 August 2017, Revised 29 September 2017, Accepted 2 October 2017

\begin{abstract}
Non-relativistic bound-energy of diatomic molecules determined by non-central potentials in five dimensional solution using AIM. Potential in five dimensional space consist of Kratzer's potential for radial part and Tangent squared potential for angular part. By varying $n_{r}, n_{1}, n_{2}, n_{3}$, dan $n_{4}$ quantum number on $\mathrm{CO}, \mathrm{NO}$, dan $\mathrm{I}_{2}$ diatomic molecules affect bounding energy values. It knows from its numerical data.
\end{abstract}

Keyword: Schrodinger equation, D-dimension, Kratzer's potential, Tangent squared potential, AIM

\section{INTRODUCTION}

Non-relativistic energy solutions can be obtained from the Schrodinger equation. The Schrodinger equation is the basis for describing a physical event related to quantum mechanics. There are many types of equations that can be used to solve the case of quantum mechanics, such as: Dirac equation (Barakat \& Alhendi, 2013), Klein-Gordon equation (Barakat, 2009), and Schrodinger equation (Arbabi, 2016). In this equation can be used interference of potentials for certain system, e.g. Poschl-Teller potential (Yahya \& Oyewumi, 2015), Deng-Fan potential and Hulthen potential (Hassanabadi et al., 2013). These equations can be applied to a higher dimension space (Dong, 2011) with the interference of a potential. Higher dimension is a space that has more than three dimensional space components.

This study was to look for energy eigenvalues on Schrodinger equations in fivedimensional space with Kratzer's potential interference and trigonometric tangen squared potential. Kratzer's potential (Bayrak et al., 2006) describes dissociation events in diatomic molecules. Kratzer's potential is used in the radial part with variable $r$. Thus, Kratzer's potential is used for the radial part

$$
V(r)=-2 D_{e}\left(\frac{a}{r}-\frac{1}{2} \frac{a^{2}}{r^{2}}\right) .
$$

where $D_{e}$ is the dissociation energy, $r$ is the distance between diatomic molecular nuclei and $a$ is the distance between the nuclei in equilibrium. Potential tangent squared 
(Ciftchi et al., 2013) describes a potential that is affected by the angular change.

Potential tangent squared at the corner with variables $\theta_{1}, \theta_{2}, \theta_{3}$, and $\theta_{4}$. The potential used for the angular parts is represented in equations (2-5).

$$
\begin{aligned}
& V\left(\theta_{1}\right)=V_{0} \tan ^{2}\left(\theta_{1}\right), \theta_{1} \in\left(-\frac{\pi}{2}, \frac{\pi}{2}\right) \\
& V\left(\theta_{2}\right)=V_{0} \tan ^{2}\left(\theta_{2}\right), \theta_{2} \in\left(-\frac{\pi}{2}, \frac{\pi}{2}\right) \\
& V\left(\theta_{3}\right)=V_{0} \tan ^{2}\left(\theta_{3}\right), \theta_{3} \in\left(-\frac{\pi}{2}, \frac{\pi}{2}\right) \\
& V\left(\theta_{4}\right)=V_{0} \tan ^{2}\left(\theta_{4}\right), \theta_{4} \in\left(-\frac{\pi}{2}, \frac{\pi}{2}\right)
\end{aligned}
$$

with $V_{0}$ is the initial potential used for each of the equally considered corner components and $\theta_{1}, \theta_{2}, \theta_{3}$, and $\theta_{4}$ are the elevation angle. This study was completed by AIM method (Falaye, 2012). In order to be solved with AIM, the Schrodinger equation is reduced to a hypergeometric type of two-order differential equation. Then the potentials in equations (1-5) are combined in form

$$
\begin{aligned}
& V\left(r, \theta_{1}, \theta_{2}, \theta_{3}, \theta_{4}\right)=-2 D_{e}\left(\frac{a}{r}-\frac{1}{2} \frac{a^{2}}{r^{2}}\right)+\frac{V_{0} \tan ^{2} \theta_{1}}{r^{2} \sin ^{2} \theta_{2} \sin ^{2} \theta_{3} \sin ^{2} \theta_{4}}+\frac{V_{0} \tan ^{2} \theta_{2}}{r^{2} \sin ^{2} \theta_{3} \sin ^{2} \theta_{4}} \\
& +\frac{V_{0} \tan ^{2} \theta_{3}}{r^{2} \sin ^{2} \theta_{4}}+\frac{V_{0} \tan ^{2} \theta_{4}}{r^{2}}
\end{aligned}
$$

with $0 \leq \theta_{1} \leq 2 \pi$ and $0 \leq \theta_{2}, \theta_{3}, \theta_{4} \leq \pi$.

\section{METHODOLOGY}

\subsection{Asymptotic Iteration Method}

From the second-order differential equation of the hypergeometric type, then a part of the differential equation which is of type AIM is taken. Two-order differential equations with type AIM is represented in equation (7) (Sari et al.,2015).

$$
y^{\prime \prime}(x)=\chi_{0}(x) y^{\prime}(x)+s_{0}(x) y(x)
$$

From equation (7) we get the values of $\chi_{0}$ and $s_{0}$, and then iteration is done with the pattern as in equations (8-9).

$$
\begin{aligned}
& \chi_{k}=\chi_{k-1}{ }^{\prime}(x)+s_{k-1}(x)+\chi_{0}(x) \chi_{k-1}(x) \\
& s_{k}=s_{k-1}{ }^{\prime}(x)+s_{0}(x) \chi_{k-1}(x)
\end{aligned}
$$

From equations (8-9) can be used to find the eigenvalues by equation (10)

$$
\Delta_{k}=s_{k}(x) \chi_{k-1}(x)-s_{k-1}(x) \chi_{k}(x)=0
$$

with $\mathrm{k}=1,2,3, \ldots$

Then to determine the eigen function of equation (7), we can use equation (11)

$$
y(x)=C_{2} \exp \left(-\int^{x} \alpha\left(x^{\prime}\right) d x^{\prime}\right)
$$

Parameter $\alpha\left(x^{\prime}\right)$ in equation (11) can be represented by equation (12). 


$$
\alpha\left(x^{\prime}\right)=\frac{s_{k}(x)}{\chi_{k}(x)}
$$

From equation (11) can be generalized to equation (13)

$$
y_{n}(x)=(-1)^{n} C_{2}(N+2)(\sigma)_{n}{ }_{2} F_{1}\left(-n, p+n, \sigma, b x^{N+2}\right)
$$

Some parameter in equation (13) is described by equations (14-15).

$$
\begin{aligned}
& (\sigma)_{n}=\frac{\Gamma(\sigma+n)}{\Gamma(\sigma)}, \sigma=\frac{2 c+N+3}{N+2}, p=\frac{(2 c+1) b+2 t}{(N+2) b} \\
& { }_{2} F_{1}\left(-n, p+n, \sigma, b x^{N+2}\right)=\sum_{n=0}^{\infty} \frac{(-n)^{n}(p+n)_{n}}{(\sigma)_{n}} \frac{\left(b x^{N+2}\right)^{n}}{n !}
\end{aligned}
$$

Where $n$ is a quantum number, $C_{2}$ is the normalization constant, ${ }_{2} F_{1}$ is a hypergeometric function. While the other parameters in equations (14-15) are obtained by comparing equation (7) with equation (16) as follows

$$
y^{\prime \prime}(x)=2\left(\frac{t x^{N+1}}{1-b x^{N+2}}-\frac{c+1}{x}\right) y^{\prime}(x)-\frac{W x^{N}}{1-b x^{N+2}}
$$

\section{SOLUTION AND DISCUSSION}

\subsection{Variable Separation}

Schrodinger equation by using natural unit $(\hbar=c=1)$ and in five-dimensional space, can be written by equation (17)

$$
\nabla_{5}^{2} \Psi+2 m(E-V) \Psi=0
$$

In equation (17) there is a Laplacian at five-dimensional coordinates. The Laplacian can be written to be

$$
\begin{aligned}
& \nabla_{5}^{2}=\frac{1}{r^{4}} \frac{\partial}{\partial r}\left(r^{4} \frac{\partial}{\partial r}\right)+\frac{1}{r^{2}}\left[\frac{1}{\sin ^{2} \theta_{2} \sin ^{2} \theta_{3} \sin ^{2} \theta_{4}} \frac{\partial^{2}}{\partial^{2} \theta_{1}}+\frac{1}{\sin ^{2} \theta_{3} \sin ^{2} \theta_{4}}\left\{\frac{1}{\sin \theta_{2}}\left(\frac{\partial}{\partial \theta_{2}} \sin \theta_{2} \frac{\partial}{\partial \theta_{2}}\right)\right\}\right. \\
& \left.+\frac{1}{\sin ^{2} \theta_{4}}\left\{\frac{1}{\sin ^{2} \theta_{3}} \frac{\partial}{\partial \theta_{3}}\left(\sin ^{2} \theta_{3} \frac{\partial}{\partial \theta_{3}}\right)\right\}\right]+\frac{1}{r^{2}}\left(\frac{1}{\sin ^{3} \theta_{4}} \frac{\partial}{\partial \theta_{4}}\left(\sin ^{3} \theta_{4} \frac{\partial}{\partial \theta_{4}}\right)\right)
\end{aligned}
$$

In equation (17) there is also a variable $\Psi$ applied in five dimensions to

$$
\Psi\left(r, \theta_{1}, \theta_{2}, \theta_{3}, \theta_{4}\right)=R(r) P_{1}\left(\theta_{1}\right) P_{2}\left(\theta_{2}\right) P_{3}\left(\theta_{3}\right) P_{4}\left(\theta_{4}\right),
$$

Then equations (18-19) are substituted into equation (17) to derive a Schrodinger equation for the radial, angular $\theta_{1}, \theta_{2}, \theta_{3}$, and $\theta_{4}$.

From combining equations (17-19), we get the five dimensional Schrodinger equation. It can be separated into equations (20-24)

$$
\begin{aligned}
& \frac{1}{r^{2}} \frac{\partial}{\partial \mathrm{r}}\left(r^{4} \frac{\partial R}{\partial \mathrm{r}}\right)+4 m r^{2} R D_{e}\left(\frac{a}{r}-\frac{1}{2} \frac{a^{2}}{r^{2}}\right)+2 m r^{2} R E+\lambda R=0 \\
& \left(\frac{\partial^{2} P_{1}}{\partial^{2} \theta_{1}}\right)-\left(\frac{2 m}{\hbar^{2}} V_{0} \tan ^{2} \theta_{1}+\lambda_{1}\right) P_{1}=0
\end{aligned}
$$




$$
\begin{aligned}
& \frac{1}{\sin \theta_{2}} \frac{\partial}{\partial \theta_{2}}\left(\sin \theta_{2} \frac{\partial P_{2}}{\partial \theta_{2}}\right)-\left(\frac{2 m}{\hbar^{2}} V_{0} \tan ^{2} \theta_{2}-\lambda_{2}-\frac{\lambda_{1}}{\sin ^{2} \theta_{2}}\right) P_{2}=0 \\
& \frac{1}{\sin ^{2} \theta_{3}} \frac{\partial}{\partial \theta_{3}}\left(\sin ^{2} \theta_{3} \frac{\partial P_{3}}{\partial \theta_{3}}\right)-\left(\frac{2 m}{\hbar^{2}} V_{0} \tan ^{2} \theta_{3}+\lambda_{3}+\frac{\lambda_{2}}{\sin ^{2} \theta_{3}}\right) P_{3}=0 \\
& \frac{1}{\sin ^{3} \theta_{4}} \frac{\partial}{\partial \theta_{4}}\left(\sin ^{3} \theta_{4} \frac{\partial P_{4}}{\partial \theta_{4}}\right)-\left(\frac{2 m}{\hbar^{2}} V_{0} \tan ^{2} \theta_{4}-\frac{\lambda_{3}}{\sin ^{2} \theta_{4}}-\lambda\right) P_{4}=0
\end{aligned}
$$

where $\lambda, \lambda_{1}, \lambda_{2}$, and $\lambda_{3}$ are constants of variabel separation.

\subsection{Solution of Radial Part}

The general solution for five dimensional Schrodinger equation in radial part is given by equation (20). Then use the equations (25-29) to reduce equation (20):

$$
\begin{aligned}
& R=U r^{-2} \\
& A=2 m D_{e} \\
& \varepsilon^{2}=-2 m E \\
& C=2 A a \\
& D=-\frac{1}{2}+\sqrt{\frac{9}{4}-\lambda+A a^{2}}
\end{aligned}
$$

Equations (25-29) are substituted into equation (20), so that equation (30) is obtained.

$$
\frac{\partial^{2} U}{\partial r^{2}}+\left(-\varepsilon^{2}+\frac{C}{r}-\frac{D(D+1))}{r^{2}}\right) U=0
$$

Equation (30) to be applicable in AIM, must use transformation in equation (31).

$$
U(r)=r^{D+1} \exp (-\varepsilon r) f(r)
$$

So, equation (30) transform into equation (32)

$$
f^{\prime \prime}(r)=2\left(\varepsilon-\frac{D+1}{r}\right) f^{\prime}(r)+\frac{2 \varepsilon(D+1)-C}{r} f(r)
$$

By comparing equation (32) with equation (7), we get the values of $\chi_{0}$ and $s_{0}$ for the radial part.

$$
\begin{aligned}
& \chi_{0}(r)=2\left(\varepsilon-\frac{D+1}{r}\right) \\
& s_{0}(r)=\frac{2 \varepsilon(D+1)-C}{r}
\end{aligned}
$$

Equations (33-34) are iterated by using equations (8-9) to obtain the parameter values $\chi_{1}, \chi_{2}, \chi_{3}, \ldots \chi_{k}$ and $s_{1}, s_{2}, s_{3}, \ldots s_{k}$. These parameter values are used to find $\Delta_{k}$. $\Delta_{k}$ is used to find energy, so we get the energy equation

$$
E_{n_{r}}=\frac{-\frac{2 m}{\hbar^{2}} D_{e}{ }^{2} a^{2}}{\left(n_{r}+\frac{1}{2}+\sqrt{\frac{9}{4}-\lambda+\frac{2 m}{\hbar^{2}} D_{e} a^{2}}\right)^{2}}
$$


The energy eigenvalue depends on the parameters of all components of the composed potential and also depend on the quantum number $n_{r}$. It can be explained by equation (35).

Then we find the wave function for radial part by using equations (11-12). So, we can resulting the ground state wave function that shown in equation (36).

$$
R_{0}(r)=r^{D-1} \exp (-\varepsilon r)
$$

and by raising parameter $k$ in equation (12), we can get the first excited wave function

$$
R_{1}(r)=(2 D+2)(D+2) r^{D-1}\left(1-\frac{2 \varepsilon_{1 D} r}{2 D+2}\right) \exp (-\varepsilon r)
$$

\subsection{Solution of Angular Part}

For reducting equation (21), we set $\tan \theta_{1}=\left(1-2 z_{1}\right) i$ and resulting equation (38)

$$
\left(z_{1}-z_{1}^{2}\right) \frac{\partial^{2} P_{1}}{\partial z_{1}{ }^{2}}+\left(1-2 z_{1}\right) \frac{\partial P_{1}}{\partial z_{1}}-\left(\frac{A_{1}}{4}+\frac{-\frac{\lambda_{1}}{4}+\frac{A_{1}}{16}}{z_{1}}+\frac{\frac{\lambda_{1}}{4}-\frac{A_{1}}{16}}{z_{1}-1}+\right) P_{1}=0
$$

we can use transformation in equations (39-41)

$$
\begin{aligned}
& P_{1}\left(\theta_{1}\right)=\left(z_{1}\right)^{\delta_{1}}\left(1-z_{1}\right)^{\gamma_{1}} f_{n}\left(z_{1}\right) \\
& \delta_{1}=\frac{1}{2} \sqrt{-\lambda_{1}+\frac{A_{1}}{4}} \\
& \gamma_{1}=\frac{1}{2} \sqrt{-\lambda_{1}+\frac{A_{1}}{4}}
\end{aligned}
$$

So, equation (21) transform into equation (42)

$$
f_{n}^{\prime \prime}\left(z_{1}\right)=\left(\frac{2 z_{1}\left(1+\delta_{1}+\gamma_{1}\right)-2 \delta_{1}-1}{z_{1}\left(1-z_{1}\right)}\right) f_{n}{ }^{\prime}\left(z_{1}\right)+\frac{\left(\delta_{1}+\gamma_{1}\right)\left(\delta_{1}+\gamma_{1}+1\right)+\frac{A_{1}}{4}}{z_{1}\left(1-z_{1}\right)} f_{n}\left(z_{1}\right)
$$

By solving equation (42) using AIM, we have the ground state wave function in equation (43) and the constant $\lambda_{1}$ in equation (44).

$$
\begin{aligned}
& P_{10}\left(\theta_{1}\right)=C_{1}\left(\frac{1+i \tan \theta_{1}}{2}\right)^{\delta_{1}+\gamma_{1}} \\
& \lambda_{1}=\frac{2 m}{\hbar^{2}} V_{0}+\left(-n_{1}-\frac{1}{2}+\sqrt{\frac{1}{4}-\frac{2 m}{\hbar^{2}} V_{0}}\right)^{2}
\end{aligned}
$$

By using $P_{2}=Q_{2}\left(\sin \theta_{2}\right)^{-\frac{1}{2}}$ in equation (22), $P_{3}=Q_{3}\left(\sin \theta_{3}\right)^{-1}$ in equation (23), and $P_{4}=Q_{4}\left(\sin \theta_{4}\right)^{-\frac{3}{2}}$ in equation (24), we can rewrite equations (22-24) the angular part equations below:

$$
Q_{2}{ }^{\prime \prime}+\left(\frac{1}{2}+\lambda_{2}+\frac{1}{4} \cot ^{2} \theta_{2}-\frac{2 m}{\hbar^{2}} V_{0} \tan ^{2} \theta_{2}+\frac{\lambda_{1}}{\sin ^{2} \theta_{2}}\right) Q_{2}=0
$$




$$
\begin{aligned}
& Q_{3}^{\prime \prime}-\left(\frac{2 m}{\hbar^{2}} V_{0} \tan ^{2} \theta_{3}+\lambda_{3}-1+\frac{\lambda_{2}}{\sin ^{2} \theta_{3}}\right) Q_{3}=0 \\
& Q_{4}{ }^{\prime \prime}+\left(\frac{3}{2}-\frac{3}{4} \cot ^{2} \theta_{4}-\frac{2 m}{\hbar^{2}} V_{0} \tan ^{2} \theta_{4}+\frac{\lambda_{3}}{\sin ^{2} \theta_{4}}+\lambda\right) Q_{4}=0
\end{aligned}
$$

By setting $\cos ^{2} \theta_{\ell}=z_{\ell}$ in equations (45-47), we obtain

$$
\begin{aligned}
& z_{2}\left(1-z_{2}\right) Q_{2}^{\prime \prime}+\frac{1}{2}\left(1-2 z_{2}\right) Q_{2}^{\prime}-\left(\frac{1+4 \lambda_{2}}{16}+\frac{m V_{0}}{2 \hbar^{2}}-\frac{m V_{0}}{2 \hbar^{2} z_{2}}+\frac{1+4 \lambda_{1}}{16\left(1-z_{2}\right)}\right) Q_{2}=0 \\
& -4\left(z_{3}-z_{3}^{2}\right) Q_{3}^{\prime \prime}-2\left(1-2 z_{3}\right) Q_{3}^{\prime}-\left(\frac{2 m}{\hbar^{2}} V_{0}\left(-1+\frac{1}{z_{3}}\right)+\lambda_{3}-1+\frac{\lambda_{2}}{1-z_{3}}\right) Q_{3}=0 \\
& \left(z_{4}-z_{4}^{2}\right) Q_{4}^{\prime \prime}+\left(\frac{1}{2}-z_{4}\right) Q_{4}^{\prime}-\frac{1}{4}\left\{\frac{9}{4}+\frac{2 m}{\hbar^{2}} V_{0}-\frac{2 m V_{0}}{\hbar^{2} z_{4}}+\frac{\lambda_{3}-\frac{3}{4}}{1-z_{4}}+\lambda\right\} Q_{4}=0
\end{aligned}
$$

Then we use transformation like equation (39) to equations (48-50). So, equations (4850) become

$$
\begin{aligned}
& f_{n}^{\prime \prime}\left(z_{2}\right)=\left(\frac{z_{2}\left(2 \delta_{2}+2 \gamma_{2}+1\right)-2 \delta_{2}-\frac{1}{2}}{z_{2}\left(1-z_{2}\right)}\right) f_{n}^{\prime}\left(z_{2}\right)+\left(\frac{\left(\delta_{2}+\gamma_{2}\right)^{2}+\frac{1+A_{2}+4 \lambda_{2}}{16}}{z_{2}\left(1-z_{2}\right)}\right) f_{n}\left(z_{2}\right) \\
& f_{n}^{\prime \prime}\left(z_{3}\right)=\frac{z_{3}\left(2 \delta_{3}+2 \gamma_{3}+1\right)-2 \delta_{3}-\frac{1}{2}}{z_{3}\left(1-z_{3}\right)} f_{n}^{\prime}\left(z_{3}\right)+\frac{\left(\delta_{3}+\gamma_{3}\right)^{2}+\frac{A_{3}+1-\lambda_{3}}{4}}{z_{3}\left(1-z_{3}\right)} f_{n}\left(z_{3}\right) \\
& f_{n}^{\prime \prime}\left(z_{4}\right)=\frac{z_{4}\left(2 \delta_{4}+2 \gamma_{4}+1\right)-2 \delta_{4}-\frac{1}{2}}{z_{4}\left(1-z_{4}\right)} f_{n}^{\prime}\left(z_{4}\right)+\frac{\left(\delta_{4}+\gamma_{4}\right)^{2}+\frac{9}{16}+A_{4}+\lambda}{z_{4}\left(1-z_{4}\right)} f_{n}\left(z_{4}\right)
\end{aligned}
$$

Parameter $\delta_{2}, \gamma_{2}, \delta_{3}, \gamma_{3}, \delta_{4}$ and $\gamma_{4}$ is described by equations (54-59).

$$
\begin{aligned}
& \delta_{2}=\frac{1}{2}+\frac{1}{2 \hbar} \sqrt{\hbar^{2}-2 m V_{0}} \\
& \gamma_{2}=\frac{1}{2}+\frac{1}{4} \sqrt{5+4 \lambda_{1}} \\
& \delta_{3}=\frac{1}{4}+\frac{1}{4} \sqrt{1-4 A_{3}} \\
& \gamma_{3}=\frac{1}{4}+\frac{1}{4} \sqrt{1-4 \lambda_{2}} \\
& \delta_{4}=\frac{1}{2}+\frac{1}{2} i \sqrt{1+4 A_{4}} \\
& \gamma_{4}=\frac{1}{16}+\frac{1}{4} \sqrt{16 \lambda_{3}-2}
\end{aligned}
$$

By equation (51-53), we can find the lowest level wave functions in equations (60-62) and the constant $\lambda_{2}, \lambda_{3}$, and $\lambda$ in equation (63-65). 


$$
\begin{aligned}
& P_{20}=C_{2}\left\{\left(\sin \theta_{2}\right)^{2 \gamma_{2}-\frac{1}{2}}-\left(\sin \theta_{2}\right)^{2\left(\delta_{2}+\gamma_{2}\right)-\frac{1}{2}}\right\} \\
& P_{30}=C_{3}\left\{\left(\sin \theta_{3}\right)^{2 \gamma_{3}-1}-\left(\sin \theta_{3}\right)^{2\left(\delta_{3}+\gamma_{3}\right)-1}\right\} \\
& P_{40}=C_{4}\left\{\left(\sin \theta_{4}\right)^{2 \gamma_{4}-\frac{3}{2}}-\left(\sin \theta_{4}\right)^{2\left(\delta_{4}+\gamma_{4}\right)-\frac{3}{2}}\right\} \\
& \lambda_{2}=\frac{1}{4}+\frac{2 m}{\hbar^{2}} V_{0}+\frac{1}{4}\left(4 n_{2}+4+2 \sqrt{1-\frac{2 m V_{0}}{\hbar^{2}}}+\sqrt{5+4 \lambda_{1}}\right)^{2} \\
& \lambda_{3}=\frac{2 m}{\hbar^{2}} V_{0}+1-\left(2 n_{3}+1+\frac{1}{2}\left(\sqrt{1-\frac{8 m}{\hbar^{2}} V_{0}}+\sqrt{1-4 \lambda_{2}}\right)\right)^{2} \\
& \lambda=-\frac{9}{16}-\frac{2 m}{\hbar^{2}} V_{0}+\left(2 i \sqrt{1+\frac{8 m}{\hbar^{2}} V_{0}}+\frac{1}{4} \sqrt{16 \lambda_{3}-2}+n_{4}+\frac{9}{16}\right)^{2}
\end{aligned}
$$

The lowest total wavefunction from equation (36), equation (43), and equation (60-62) is given as

$$
\begin{aligned}
& \Psi_{00000}=C_{g a b} r^{D-1} \exp (-\varepsilon r)\left(\frac{1+i \tan \theta_{1}}{2}\right)^{\delta_{1}+\gamma_{1}}\left\{\left(\sin \theta_{2}\right)^{2 \gamma_{2}-\frac{1}{2}}-\left(\sin \theta_{2}\right)^{2\left(\delta_{2}+\gamma_{2}\right)-\frac{1}{2}}\right\} \\
& \left\{\left(\sin \theta_{3}\right)^{2 \gamma_{3}-1}-\left(\sin \theta_{3}\right)^{2\left(\delta_{3}+\gamma_{3}\right)-1}\right\}\left\{\left(\sin \theta_{4}\right)^{2 \gamma_{4}-\frac{3}{2}}-\left(\sin \theta_{4}\right)^{2\left(\delta_{4}+\gamma_{4}\right)-\frac{3}{2}}\right\}
\end{aligned}
$$

with $C_{g a b}=C_{1} C_{2} C_{3} C_{4}$ which is normalization factor of the total lowest wave function.

\subsection{Discussion}

Eigen value of energy in equation (35), and constants of variabel separation in equation (44) and equations (63-65) can calculated by using computational method. We can calculate numerical solution of the energy for diatomic molekul are listed in Table 1. From Table 1 it can be seen if $\mathrm{CO}$ has greater dissociation energy than $\mathrm{NO}$ and $\mathrm{I}_{2}$. But $\mathrm{CO}$ has the lowest mass and equilibrium distance of nucleus. From parameters in Table 1 , numerical solution of energy are shown in Table 2 . The negative value show that the energy is repulsive. Repulsive energy of CO and NO molecules decrease caused by the increase of quantum number $n_{4}$ is more significant than the increase in energy caused by the increase of the quantum number $n_{r}$ and the decrease of energy due to the increase in $n_{1}, n_{2}, n_{3}$. But for the diatomic molecule $\mathrm{I}_{2}$, the increase of all quantum numbers causes the value of the repulsive energy getting smaller.

Table 1. Mass and Spectroscopic Properties of Diatomic Molecular Variations

\begin{tabular}{llll}
\hline \multicolumn{1}{c}{ Parameter } & \multicolumn{1}{c}{$\mathrm{CO}$} & \multicolumn{1}{c}{$\mathrm{NO}$} & \multicolumn{1}{c}{$\mathrm{I}_{2}$} \\
\hline$D_{e}(\mathrm{eV})$ & 10.84514471 & 8.043782568 & 1.581791863 \\
$a\left(\mathrm{eV}^{-1}\right)$ & $5.7174 \times 10^{-4}$ & $5.8319 \times 10^{-4}$ & $13.000 \times 10^{-4}$ \\
$m(\mathrm{eV})$ & $6.3904 \times 10^{9}$ & $6.9566 \times 10^{9}$ & $59.104 \times 10^{9}$ \\
\hline
\end{tabular}


Table 2. The Energy Spectrum of Particles

\begin{tabular}{lllllrrr}
\hline \multirow{2}{*}{$\mathrm{n}_{\mathrm{r}}$} & $\mathrm{n}_{1}$ & $\mathrm{n}_{2}$ & $\mathrm{n}_{3}$ & $\mathrm{n}_{4}$ & \multicolumn{3}{c}{$\mathrm{CO}(\mathrm{eV})$} \\
\cline { 6 - 8 } & & & & & 0 & $\mathrm{NO}$ & $\mathrm{I}_{2}$ \\
\hline 0 & 0 & 0 & 0 & 0 & $-0,620569$ & $-0,316085$ & $-0,1237857$ \\
2 & 0 & 0 & 0 & 0 & $-0,624640$ & $-0,318575$ & $-0,1237739$ \\
0 & 1 & 0 & 0 & 0 & $-0,628649$ & $-0,321032$ & $-0,1237617$ \\
0 & 2 & 0 & 0 & 0 & $-0,620562$ & $-0,316081$ & $-0,1237856$ \\
0 & 0 & 1 & 0 & 0 & $-0,620556$ & $-0,316078$ & $-0,1237854$ \\
0 & 0 & 2 & 0 & 0 & $-0,620542$ & $-0,316071$ & $-0,1237852$ \\
0 & 0 & 0 & 1 & 0 & $-0,616316$ & $-0,312961$ & $-0,1238835$ \\
0 & 0 & 0 & 2 & 0 & $-0,611919$ & $-0,309746$ & $-0,1239813$ \\
0 & 0 & 0 & 0 & 1 & $-0,610426$ & $-0,310584$ & $-0,1234873$ \\
0 & 0 & 0 & 0 & 2 & $-0,600325$ & $-0,305110$ & $-0,1231891$ \\
\hline
\end{tabular}

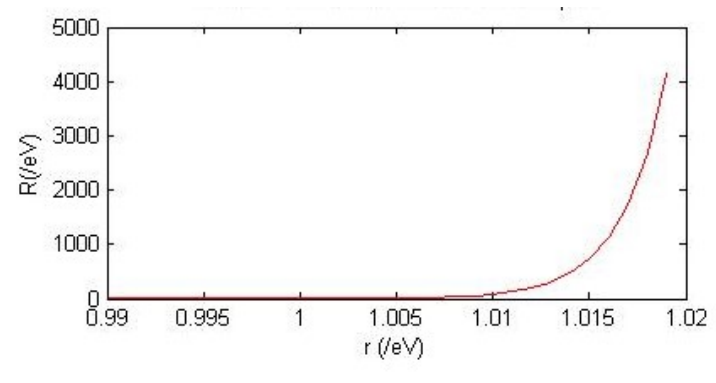

(a)

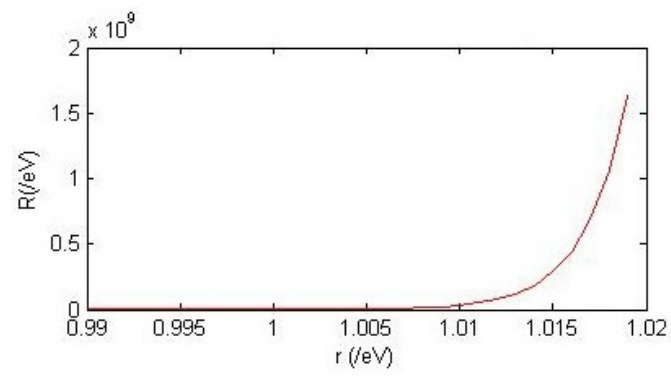

(b)

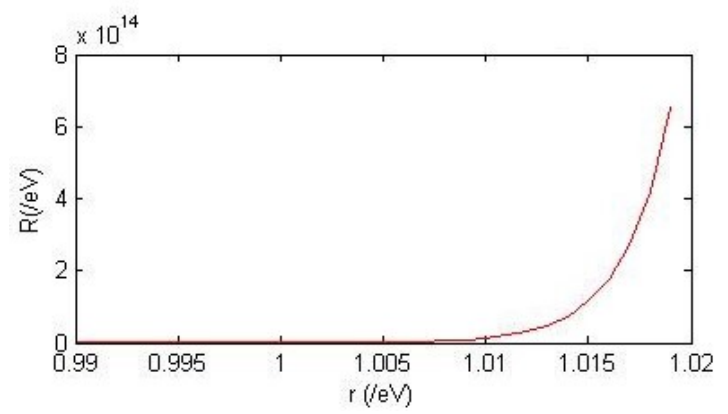

(c)

Figure 1. Radial wave function with variation $n_{r}$ : (a) $n_{r}=0$, (b) $n_{r}=1$, (c) $n_{r}=2$

Figure (1) is ilustration of equation (36). At certain ranges the wave function looks constant and then at certain values the wave function toward infinity. When at a certain $r$ value, $n_{r}=2$ has larger wave function than $n_{r}=0$ and $n_{r}=1$. If quantum number $n_{r}$ is getting greater, the wave function getting greater too. 


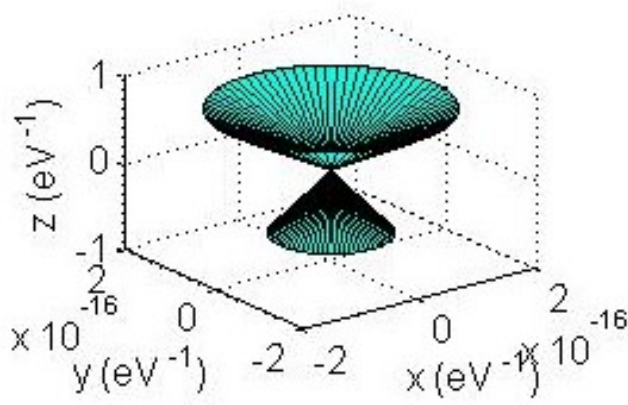

(a)

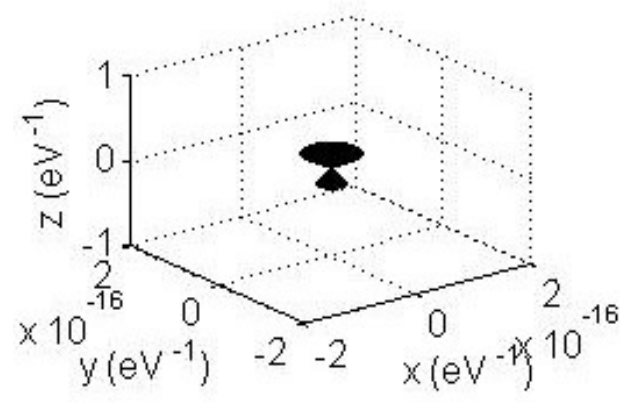

(b)

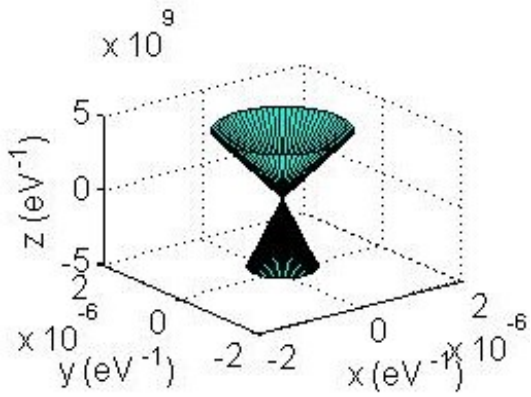

(c)

Figure 2. Wave function $P_{1}$ on spherical coordinates with varying $n_{1}$ : (a) $n_{1}=0$,

$$
\text { (b) } n_{1}=1 \text {, (c) } n_{1}=2 \text {. }
$$

Equation (43) is portrayed by Figure 2. It shows that the increase of the quantum number $n_{1}$ affected to wave function. However the wave function of the quantum number $n_{1}=0$ is greater than wave function of the quantum number $n_{1}=1$, wave function of the quantum number $n_{1}=2$ has the greatest of them. The shape of wave function in Figure (2) is faced-cone. The upper cone is bigger than a lower cone. This shape is represented a probability of diatomic molecule distribution.

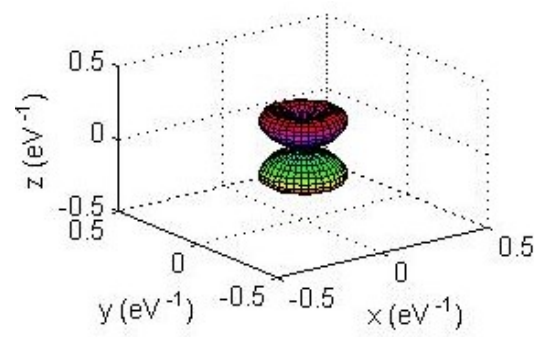

(a)

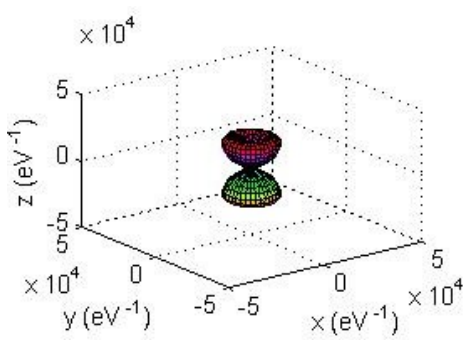

(b)

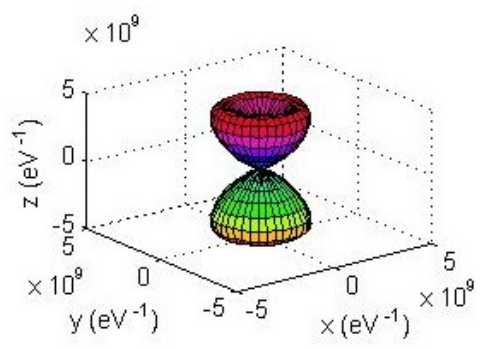

(c)

Figure 3. Wave function $P_{2}$ on spherical coordinates with varying $n_{2}$ : (a) $n_{2}=0$, (b)

$$
n_{2}=1 \text {, (c) } n_{2}=2 \text {. }
$$




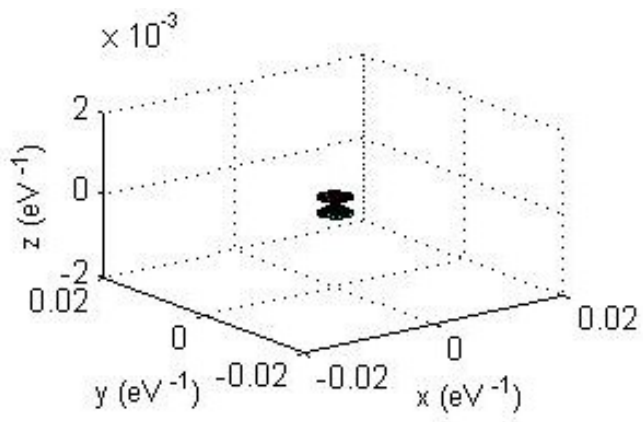

(a)

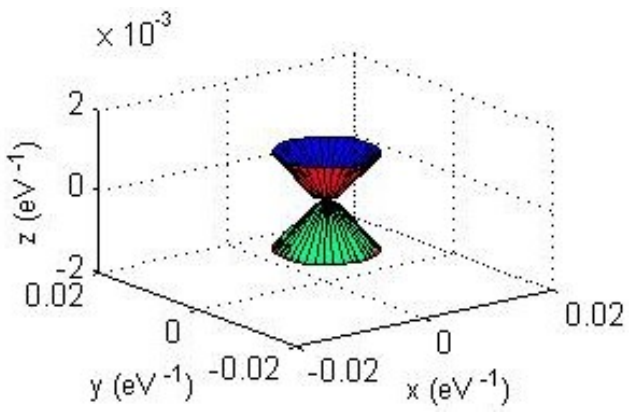

(b)

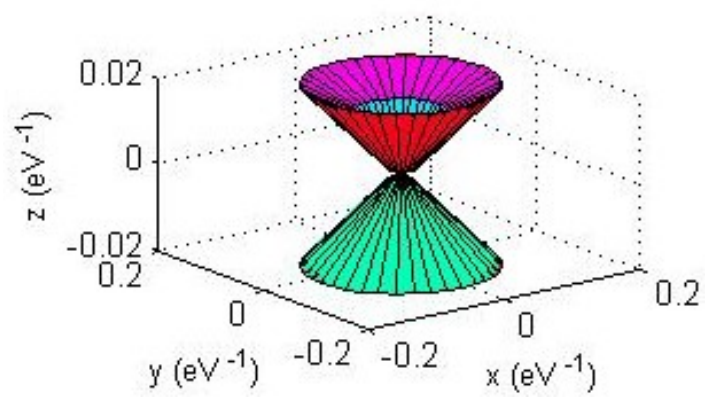

(c)

Figure 4. Wave function $P_{3}$ on spherical coordinates with varying $n_{3}$ : (a) $n_{3}=0$, (b) $n_{3}=1$, (c) $n_{3}=2$.

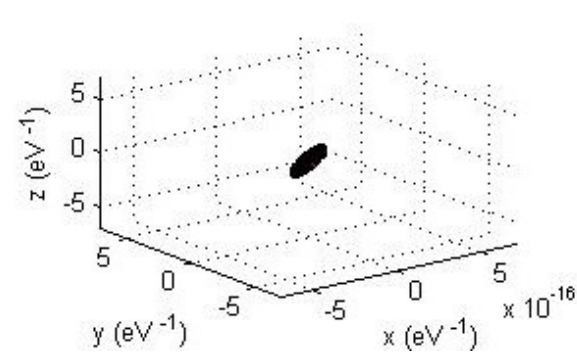

(a)

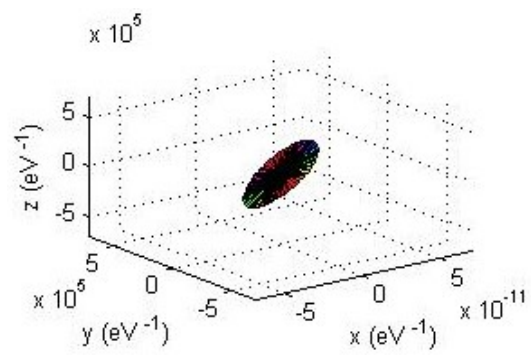

(b)

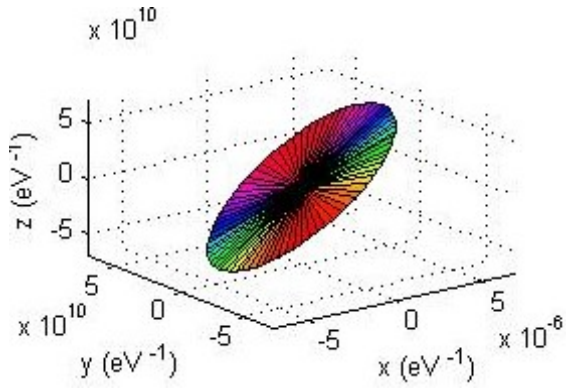

(c)

Figure 5. Wave function $P_{4}$ on spherical coordinates with varying $n_{4}$ : (a) $n_{4}=0$, (b)

$$
n_{4}=1, \text { (c) } n_{4}=2 \text {. }
$$

Equation (60) portrayed by Figure (3), equation (61) portrayed by Figure (4), and equation (62) portrayed by Figure (5). All of them show that increasing own quantum number make an increasing own wave function. But all of them have a different at own 
shape: Figure (3) is faced half-spheris, Figure (4) is faced-cone and Figure (5) is tilted circle. Their shape is affected by their wave function.

\section{CONCLUSION}

In this paper, we have presented the solution of five dimensional Schrodinger for some diatomic molecule with disturbance Kratzer's potential combined with trigonometric tangent squared potential used AIM. We have gotten eigen value of energy total from solution radial part. In eigen value energy, there are constants of variabel separation. It is resulted from all angular part. So, the eigen value energy depended on all quantum number. The radial wavefunctions and angular wavefunctions was obtained using wave function generator in equation (11) or equation (13). After we have had an eigen value energy and all wave function, we can result numerical solution for eigen value energy and plotting wave function in spherical coordinates.

\section{FUTURE WORKS}

Suggestions can be given for further research:

a. The variable equations used to reduce Schrodinger equations at the angle are made equal, so there is no difference in the shape of the probability of finding a particle or a diatomic molecule.

b. More understanding of quantum mechanics, particle physics and the concept of plotting real and imaginary waves.

c. Need to do research with the same case, but different methods to be able to compare energy value and wave function.

\section{Acknowledgments}

This Research was partly supported by Higher Education Project Grant with contract no. $632 / \mathrm{UN} 27.21 / \mathrm{LT} / 2016$

\section{References}

Arbabi, S. (2016). Exact solitary wave solutions of the complex nonlinear Schrödinger equations. Optik, 4682-4688.

Barakat, T. (2009). Perturbed Coulomb potentials in the Klein-Gordon equation via the asymptotic iteration method. Annals of Physics, 725-733.

Barakat, T., \& Alhendi, H.A. (2013). Generalized Dirac Equation with Induced EnergyDependent Potential via Simple Similarity Transformation and Asymptotic Iteration Methods. Found Physics, 43, 1171-1181.

Bayrak, O., Boztosun, I., \& Ciftci, H. (2006). Exact Analytical Solutions to the Kratzer Potential by the Asymptotic Iteration Method. Wiley InterScience, 107, 540544.

Ciftchi, H., Hall, R.L., \& Saad, N. (2013). Exact and approximate solutions of Schrödinger's equation for a class of trigonometric potentials. Central 
European Journal of Physics, 11(1), 37-48.

Dong, Shi Hai. (2011). Wave equations in higher dimensions. New York : Springer.

Falaye, B. (2012). Arbitrary $\ell$-State Solutions of the Hyperbolical Potential by the Asymptotic Iteration Method. Few-Body Syst, 53, 557-562

Hassanabadi, M., Yazarloo, B.H., Mahmoudieh, M., \& Zarrinkamar, S. (2013). Dirac equation under the Deng-Fan potential and the Hulthen potential as a tensor interaction via SUSYQM. The European Physical Journal Plus, 128, 111-123

Sari, R.A., Suparmi, A., Cari, C. (2015). Solution of Dirac equation for Eckart potential and trigonometric Manning Rosen potential using asymptotic iteration method. Chin. Phys. B, 25

Yahya, W., \& Oyewumi, K. (2015). Thermodynamic properties and approximate solutions of the 1 state Poschl-Teller-type potential. Journal of the Association of Arab Universities for Basic and Applied Sciences. 